\title{
Role of inflammatory mediators in the suppression of insulin receptor phosphorylation in circulating mononuclear cells of obese subjects
}

\author{
H. Ghanim • A. Aljada • N. Daoud • R. Deopurkar • \\ A. Chaudhuri $\cdot$ P. Dandona
}

Received: 21 June 2006 /Revised: 25 September 2006 / Accepted: 26 September 2006 / Published online: 16 December 2006

(C) Springer-Verlag 2006

\begin{abstract}
Aims/hypothesis Obesity is associated with insulin resistance and inflammation. The circulating human mononuclear cell (MNC) has been shown to respond to low-dose insulin infusion. We have now investigated whether in obesity: (1) phosphorylated insulin receptor beta subunit (p-INSR- $\beta$ ) is reduced in the $\mathrm{MNC}$; (2) pro-inflammatory mediators including inhibitor of kappa light polypeptide gene enhancer in B cells-kinase beta (IKBKB), suppressor of cytokine signalling-3 (SOCS) and protein kinase C-beta 2 (PRKCB2) are increased and related to $\mathrm{p}-\mathrm{INSR}-\beta$; and (3) the reduction in MNC p-INSR- $\beta$ is related to the reduction in insulin sensitivity.

Materials and methods MNCs were prepared from fasting blood samples of 16 normal weight and 16 obese female subjects.

Results Our data show that $\mathrm{p}-\mathrm{INSR}-\beta$ is reduced significantly in MNCs from obese subjects compared with that of normal controls. MNCs from obese subjects have higher IKBKB expression, increased nuclear factor kappa $\mathrm{B}(\mathrm{NF} \kappa \mathrm{B})$ binding and higher mRNA expression of TNFAIPI and IL6 genes. NFKB binding, TNFAIP1 mRNA and plasma C-reactive
\end{abstract}

Electronic supplementary material Supplementary material is available in the online version of this article at http://dx.doi.org/ $10.1007 / \mathrm{s} 00125-006-0508-9$ and is accessible to authorised users.

H. Ghanim · A. Aljada • N. Daoud · R. Deopurkar •

A. Chaudhuri $\cdot$ P. Dandona

Division of Endocrinology, Diabetes and Metabolism,

State University of New York at Buffalo and Kaleida Health,

Buffalo, NY, USA

P. Dandona $(\bowtie)$

Diabetes-Endocrinology Center of Western New York,

3 Gates Circle,

Buffalo, NY 14209, USA

e-mail: pdandona@kaleidahealth.org protein are inversely related to $\mathrm{p}-\mathrm{INSR}-\beta$. PRKCB $2 \mathrm{mRNA}$ and protein expression were significantly higher in the obese subjects and were related significantly to pro-inflammatory mediators but not to p-INSR- $\beta$. SOCS3 mRNA expression was markedly elevated and positively related to proinflammatory mediators including IKBKB and PRKCB2 on the one hand and inversely related to $p$-INSR- $\beta$ on the other.

Conclusions/interpretation We conclude that in obesity the MNC is characterised by reduced p-INSR- $\beta$ and increased inflammatory mediators including IKBKB, PRKCB2 and SOCS3. The increase in SOCS3 but not IKBKB or PRKCB2 is related inversely to $\mathrm{p}$-INSR- $\beta$ and might mediate the inhibition of $p$-INSR- $\beta$. These data elucidate the relationship between inflammation and insulin resistance using the MNC as a model.

Keywords Inflammation - Insulin receptor . Insulin resistance $\cdot$ Mononuclear cells $\cdot$ Obesity $\cdot$ SOCS3
Abbreviations
CRP C-reactive protein
EGR1 early growth response gene-1
EMSA electrophoretic mobility shift assay
HOMA-IR homeostasis model assessment of insulin resistance
IкB inhibitor kappa B
IKBKB inhibitor of kappa light polypeptide gene enhancer in B cells-kinase beta
INSR insulin receptor
INSR- $\beta \quad$ insulin receptor beta subunit
INSRS-1 insulin receptor substrate-1 and -2
and -2
JAK-STAT Janus kinase and the signal transducer and activator of transcription 
MNC mononuclear cell

NFkB nuclear factor kappa B

PI3KRA phosphoinositide-3-kinase, regulatory

subunit 1 (p85 alpha)

PI3KCA phosphoinositide-3-kinase, catalytic alpha

polypeptide (p110 alpha)

p-INSR- $\beta$ phosphorylated insulin receptor beta subunit

PRKCB2 protein kinase C-beta 2

SOCS3 suppressor of cytokine signalling-3

TNFAIP1 tumour necrosis factor-alpha

\section{Introduction}

Recent evidence points to a causal relationship between inflammation and insulin resistance. The pioneering studies from Hotamisligil and Spiegelman demonstrated that adipocytes express tumour necrosis factor-alpha (TNFAIP1, also known as $T N F \alpha$ ) constitutively and that expression increases in the obese [1-3]. Plasma concentrations of TNFAIP1 have been shown to be elevated in human obesity [4]. They also demonstrated that in adipocytes, TNFAIP1 reduces insulin receptor (INSR) tyrosine phosphorylation and INSR substrate (INSRS)-1 tyrosine phosphorylation through an increase in serine phosphorylation of INSRS-1, which in turn inhibits the tyrosine phosphorylation of the INSR. Thus, TNFAIP1 probably mediates insulin resistance in many rodent obesity models. It is overexpressed in white adipose tissue in obese humans [4].

While most of the studies have looked at insulin action and insulin resistance and its possible link to inflammation in the classic insulin target tissues, i.e. adipose tissue and skeletal muscle, little is known about insulin signalling and the insulin resistance state of other cell types that are involved in the inflammatory process, the endothelial cell and the circulating mononuclear cell (MNC). Insulin signal transduction is altered by TNFAIP1 in adipocytes. Our previous work has shown that TNFAIP1 inhibits insulin-induced tyrosine phosphorylation of INSR beta subunit (INSR- $\beta$ ) in human aortic endothelial cells, in vitro, and reduces the total amount of INSR and the expression of endothelial nitric oxide synthase [5]. We have also shown that insulin infusions suppress the pro-inflammatory nuclear factor kappa $\mathrm{B}$ (NFkB) binding activity, increase the total inhibitor kappa B (IKB) and suppress early growth response gene-1 (EGR-1) in MNCs from obese subjects $[6,7]$. These actions reflect an antiinflammatory effect of insulin on MNCs and point to the fact that the MNC is a target of insulin action. Although INSR was first demonstrated in MNCs 30 years ago [8], the biological action of insulin on this cell was not demonstrated until our recent work $[6,7]$. Hitherto, there have been no data on insulin signal transduction in this cell.
The MNC is in a pro-inflammatory state in obesity [9] and this is associated with a systemic low-grade inflammation characterised by higher TNFAIP1, interleukin-6 (IL6) and $\mathrm{C}$-reactive protein (CRP) concentrations in the plasma [10-12]. However, the interaction between insulin resistance and inflammatory mediators has not been studied in this cell population in obesity. Therefore, we embarked on a study to investigate the relationship between certain mediators of cellular inflammation which have been linked to impaired insulin signalling through the suppression of INSR phosphorylation.

Among the mediators investigated were: (1) the inhibitor of kappa light polypeptide gene enhancer in B cells-kinase beta (IKBKB)-NFKB pathway and its target genes such as TNFAIP1 and IL6; (2) the protein kinase C (PRKC) family of serine/threonine kinases, which are known to inhibit INSR and INSRS phosphorylation [13-15]; and (3) the expression of two members of the suppressor of cytokine signalling (SOCS) family, SOCS1 and SOCS3, which have been associated with impaired insulin signalling [16-18]. It is of great interest that the same proteins may also mediate resistance to leptin action, which is known to occur in human obesity.

We therefore hypothesise that in the freshly isolated MNC from obese subjects: (1) insulin signal transduction, as represented by the phosphorylated INSR- $\beta$ (p-INSR- $\beta$ ) to INSR- $\beta$ ratio, is suppressed; (2) the increase in the expression of pro-inflammatory mediators in obesity is related to the suppression of $p$-INSR- $\beta$ : INSR- $\beta$; (3) there is an increase in PRKC isoforms and SOCS expression as a part of the proinflammatory process and that this is inversely related to the p-INSR- $\beta$ :INSR- $\beta$ ratio; and (4) the changes in p-INSR- $\beta$, pINSR- $\beta$ :INSR- $\beta$ ratio, PRKC isoforms and SOCS expression are related to the homeostasis model assessment of insulin resistance (HOMA-IR) index.

\section{Subjects and methods}

Subjects Two groups, 16 each, of normal weight controls $\left(B M I=22.6 \pm 1.9 \mathrm{~kg} / \mathrm{m}^{2}\right)$ and obese $\left(B M I=40.0 \pm 4.4 \mathrm{~kg} / \mathrm{m}^{2}\right)$ female subjects were recruited for this study. The Internal Review Board of the State University of New York at Buffalo approved the study and written consent was obtained from all subjects. Fasting blood samples were collected in the morning between 0800 and 1000 hours. Subjects' demographic data are summarised in Table 1.

Measurement of plasma insulin, NEFA and pro-inflammatory mediator concentrations Insulin was measured in fasting plasma samples using an ELISA kit (Diagnostics Systems Laboratories, Inc., Webster, TX, USA). NEFA concentration was measured using the Half-Micro calori- 
metric kit from Roche Diagnostic (Indianapolis, IN, USA). Plasma TNFAIP1 and IL6 concentrations were measured with a high-sensitivity ELISA kit from R\&D Systems (Minneapolis, MN, USA). Plasma CRP was measured using an ELISA kit from Alpha Diagnostic International (San Antonio, TX, USA). HOMA-IR was calculated according to Matthews et al. [19].

MNC isolation Fasting blood samples were collected in sodium-EDTA as an anticoagulant. Four millilitres of blood sample were layered carefully over $3.5 \mathrm{ml}$ PMN medium (Robbins Scientific Corp., Sunnyvale, CA, USA). The upper layer containing the MNCs was harvested and washed with Hanks' balanced salt solution and then with PBS.

Western blotting MNC total cell lysates were prepared and western blotting performed as previously described [9]. Polyclonal or monoclonal antibodies against INSR- $\beta$, phosphoinositide-3-kinase, regulatory subunit 1 (p85 alpha) (PI3KRA), phosphoinositide-3-kinase catalytic, alpha polypeptide (p110 alpha) (PI3KCA) and IKBKB (BD Biosciences, San Jose, CA, USA), INSRS-1 (Upstate USA, Inc., Charlottesville, VA, USA), pINSR- $\beta^{\text {(Tyr1 162,Tyr1163) }}$ (EMD Biosciences, Inc., San Diego, CA, USA), protein kinase C-beta 2 (PRKCB2) and actin (Santa Cruz Biotechnology, Santa Cruz, CA, USA) were used. Two different antibodies against SOCS3 were tested (Santa Cruz Biotechnology and Immuno-Biological Laboratories Co., Tokyo, Japan). There was no detectable signal for INSRS-1 or INSRS-2 tyrosine or serine phosphorylation at basal levels. Densitometry was performed using Molecular Analyst software and all values were corrected for loading with actin.

Total RNA isolation and real-time RT-PCR Total RNA isolation and RT-PCR were performed as previously described $[9,20]$. The specificity and the size of the PCR products were tested by adding a melting curve at the end

Table 1 Summary of subject demographic data (means \pm SD)

\begin{tabular}{lll}
\hline & Lean subjects & Obese subjects \\
\hline$n$ & 16 & 16 \\
BMI & $22.6 \pm 1.9$ & $40.0 \pm 4.4^{*}$ \\
Age (years) & $36.9 \pm 11.8$ & $43.2 \pm 8.6$ \\
BP, systolic (mmHg) & $111 \pm 11.7$ & $127 \pm 22.7$ \\
BP, diastolic (mmHg) & $72.2 \pm 7.0$ & $81.1 \pm 9.4$ \\
Glucose (mmol/l) & $4.46 \pm 0.42$ & $4.64 \pm 0.46$ \\
Insulin (pmol/l) & $32.57 \pm 16.65$ & $120.04 \pm 45.49^{*}$ \\
HOMA-IR & $0.74 \pm 0.5$ & $3.15 \pm 2.0^{*}$ \\
NEFA (mmol/l) & $0.43 \pm 0.2$ & $0.59 \pm 0.1 *$ \\
Cholesterol (mmol/l) & $5.02 \pm 0.81$ & $5.25 \pm 1.14$ \\
Triacylglycerol (mmol/l) & $0.686 \pm 0.363$ & $1.334 \pm 0.701^{*}$ \\
\hline
\end{tabular}

$* p<0.05$ of the amplifications and by running on a $2 \%$ agarose gel. All values were normalised to $18 \mathrm{~S}$ expression.

$N F \kappa B$ DNA binding activity Nuclear extracts from MNCs were prepared by a high-salt extraction method [21] and used to measure nuclear NFKB DNA binding activity by an electrophoretic mobility shift assay (EMSA) as previously described [9]. Specificity of the assay was tested by incubating the samples with specific antibodies against Rel-A (H-286) and p50 (H-119) subunits of the NFKB complex (Santa Cruz Biotechnology).

Statistical analysis Statistical analysis was carried out using SigmaStat software (Systat Software, Inc., San Jose, $\mathrm{CA}$, USA). All values are means $\pm \mathrm{SE}$, unless otherwise indicated. The percentage change is calculated from the means of the groups. Statistical analysis was carried out using an unpaired $t$ test between normal weight control subjects and obese subjects. Correlation analysis was performed using Spearman rank order correlation between p-INSR- $\beta$ and pro-inflammatory markers. The sample size for correlation analysis was 26 , which represents the number of samples from both obese and normal weight subjects that were analysed for all the parameters.

\section{Results}

RNA expression of insulin signalling mediator In the obese subjects the expression of INSR was significantly higher by $52.5 \% \quad(p=0.032), I N S R S-2$ by $45.6 \%(p=0.033)$ and PI3KCA (PI3K-p110) by $43.3 \%(p=0.032)$, and was significantly lower for $A K T 2$ by $20.0 \%$ ( $p=0.036$ ), as shown in Fig. 1. There was no significant difference in the mRNA expression levels of INSRS-1, PI3KRA (PI3K-p85) and $A K T 2$ between the two groups (Fig. 1).

Protein content of insulin signalling mediators Total protein content of INSR- $\beta$, INSRS-1, PI3KRA and PI3KCA in the obese individuals was not significantly different from that in the normal weight (Electronic supplementary material [ESM] Fig. 1). INSRS-2 protein was not detectable when the membranes were probed with either a polyclonal or monoclonal antibody against INSRS-2.

INSR tyrosine phosphorylation The INSR- $\beta$ phosphorylation at tyrosine 1,162 and tyrosine 1,163 (p-INSR- $\beta$ ) is required for the tyrosine kinase activity of the INSR. When these sites were probed with an affinity-purified specific polyclonal antibody, $\mathrm{p}$-INSR- $\beta$ was significantly lower (by $31 \%)$ in the obese subjects $(p=0.019)$ compared with that of lean subjects. in addition, $\mathrm{p}$-INSR- $\beta$ was significantly 


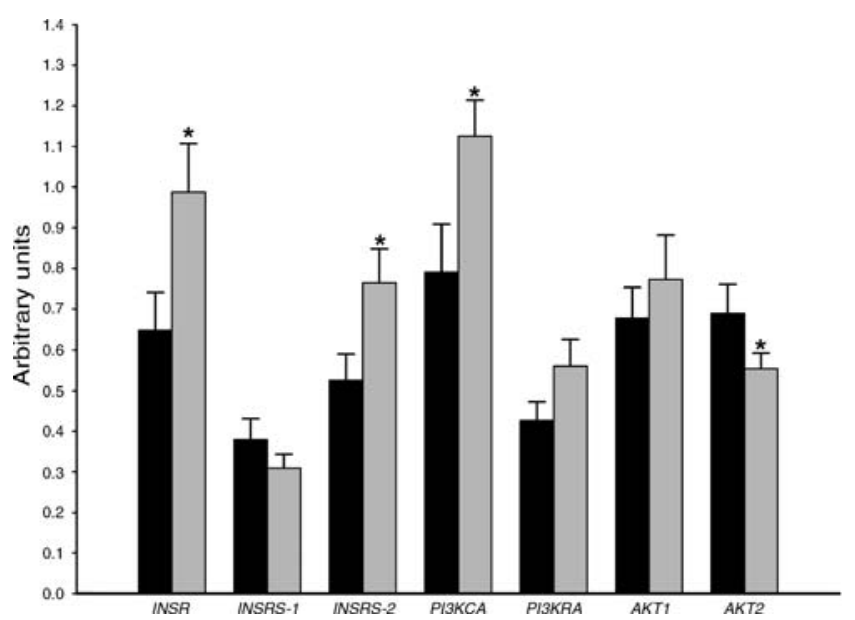

Fig. 1 Gene expression of insulin signalling mediators. Total RNA was isolated from MNCs from obese subjects (grey bars) and lean subjects (black bars) and gene expression measured by real-time RTPCR. Values are normalised to $18 \mathrm{~S}$ expression $(n=16$ vs 16$) .{ }^{*} p<0.05$

lower by $33 \%(0.49 \pm 0.2$ vs $0.74 \pm 0.25, p=0.008)$ in the obese subjects when measured as the ratio of p-INSR$\beta^{\text {(Tyr1162,Tyr1 163) }}$ to total INSR- $\beta$ (Fig. 2). These values were obtained by stripping the blots and reprobing with a polyclonal antibody against INSR- $\beta$. There was no detectable signal for basal INSRS-1 tyrosine or serine phosphorylation after probing with two different antibodies for these phosphorylation sites.

Levels of pro-inflammatory mediators $\mathrm{NFKB}$ binding activity in the MNCs from the obese subjects was significantly higher by $28.3 \%(p<0.05$, Table 2, ESM Fig. 2$)$. $I K B K B$ mRNA expression in the MNC was significantly higher by $45 \%(p<0.05$, Fig. 3$)$ in the obese than the lean subjects, while IKBKB protein production was also higher (by $25 \%$ ) in the obese group but was not statistical significant $(p<0.085$, Fig. 3). TNFAIP1 and IL6 mRNA expression were significantly higher by $72 \%(p=0.04)$ and by $129 \%(p=0.002)$, respectively (Table 2$)$ in the obese subjects than in the normal control subjects. Circulating levels of IL6 were higher by $124 \%$ (3.297 \pm 0.895 vs $1.445 \pm$ $0.423 \mathrm{pg} / \mathrm{ml}, p<0.001)$, TNFAIP 1 by $31 \%(3.753 \pm$ $0.621 \mathrm{pg} / \mathrm{ml}$ vs $2.772 \pm 0.311 \mathrm{pg} / \mathrm{ml}, p=0.014)$ and CRP by $304 \%(3,041 \pm 654 \mathrm{ng} / \mathrm{ml}$ vs $843 \pm 125 \mathrm{ng} / \mathrm{ml}, p<0.001)$ in the obese group (Table 2).

$R N A$ and protein levels of PRKC isoforms The PRKCB2 mRNA expression was significantly higher in the obese subjects by $81 \%(p=0.002)$ as shown in Fig. 4 a. PRKCB2 protein content was significantly higher by $41 \%(p<0.05)$ in the obese subjects than in normal lean subjects (Fig. 4b). Obese subjects had slightly but not significantly higher
mRNA expression of $P R K C B 1, P R K C Q$ and $P R K C Z$ isoforms.

RNA expression of SOCS1 and SOCS3 In obese subjects, the expression of SOCS3 mRNA was significantly greater by $219 \%(p<0.001)$ as shown in Fig. 5. There was no significant difference in the mRNA expression of SOCS1 between the two groups (Fig. 5). We were unable to detect a quantifiable signal of SOCS3 when two different antibodies were tested by western blotting.

Correlation analysis A Spearman rank order correlation coefficient was calculated for the combined data from both groups to assess the relationship between p-INSR- $\beta$ and proinflammatory mediators. Tyrosine phosphorylation of the INSR was inversely related to TNFAIP1 mRNA expression $(r=-0.616, p<0.001)$ and plasma CRP levels $(r=-0.481$, $p=0.013$ ) (ESM Fig. 3a,b). There was an inverse relationship between $p$-INSR- $\beta$ : INSR- $\beta$ ratio and NFKB binding activity $(r=-0.526, p=0.006$, ESM Fig. 3c). Plasma TNFAIP1 and IL6 concentrations also showed a trend towards an inverse relationship with p-INSR- $\beta$ ( $r=-0.321$, $p=0.10$ and $r=-0.307, p=0.12$, respectively). There was a positive relationship between SOCS3 mRNA and all of the following: BMI, HOMA-IR, NFKB binding (ESM Fig. 3d,e), mRNA expression of TNFAIPI and IL6 and plasma NEFA and CRP levels. SOCS3 mRNA was inversely related to both p-INSR- $\beta$ (ESM Fig. $3 f$ ) and the p-INSR- $\beta$ : INSR- $\beta$ ratio. In addition, both $P R K C B 2$ mRNA and PRKCB2 protein levels were significantly related to BMI and NEFA and CRP levels. In addition, PRKCB2 mRNA showed a significant relationship to NFKB binding and IL6 mRNA, while it was not related to HOMA-IR, $\mathrm{p}$-INSR- $\beta$ or $\mathrm{p}-\mathrm{INSR}-\beta$ : INSR- $\beta$ ratio. PRKCB2 protein levels were also related positively to TNFAIP1 mRNA and plasma TNFAIP1 and IL6 concentrations, while there was only a trend towards a negative correlation with $p$-INSR- $\beta$ and $p$-INSR- $\beta$ : INSR- $\beta$ and a trend towards a positive correlation with HOMA-IR index. All correlations are summarised in ESM Table 1.

\section{Discussion}

Our data show that there is an increase in the MNC of the obese of three putative candidate molecules which may participate in the pathogenesis of insulin resistance: IKBKB, PRKCB2 and SOCS3. The increases in IKBKB and PRKCB2 were observed at both the protein and mRNA levels, while the increase in SOCS3 was observed only at the mRNA level. Repeated attempts to demonstrate a quantifiable SOCS3 protein in MNC failed, probably due to the small amounts of protein contained in the cellular 


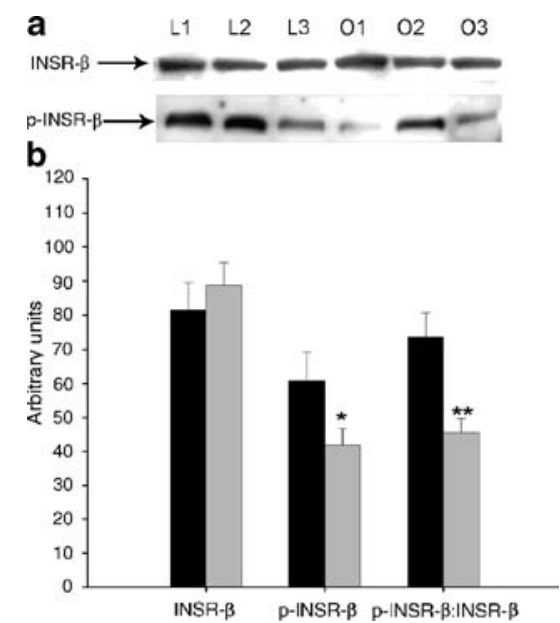

Fig. 2 INSR- $\beta$ and $p$-INSR- $\beta$ protein quantification by western blotting. a Protein content of INSR- $\beta$ subunit and $p$-INSR- $\beta$ subunit from total cell lysates of three lean $(L)$ and three obese $(O)$ subjects. b Densitometry of protein content of INSR- $\beta$ and p-INSR- $\beta$ and the calculated ratio of p-INSR- $\beta$ : INSR- $\beta$ (multiplied by 100 for scale purposes) ( $n=16$ vs 16$)$. Grey bars, obese subjects; black bars, lean subjects. ${ }^{*} p<0.05 ;{ }^{*} p<0.01$

homogenates and the limitation of the technique. A faint, inconsistent band at the target size of SOCS3 was often observed, but it was not sufficient to evaluate the protein production. SOCS3 protein is unstable and very sensitive to proteasomal degradation [22], which is a robust process in this cell type, and we may require additional specific inhibitor(s) to block this process. Sensitive proteomic techniques may have to be employed to elucidate this issue. It is also possible that viable SOCS3 protein may not be produced in MNCs due to post-transcriptional inhibitors or modifications and that mRNA levels do not reflect changes at the protein level. Therefore, SOCS3 mRNA data should be considered with caution until further protein data are available.

Both PRKCB2 and SOCS3 mRNA were related significantly to a majority of inflammatory indices, both intracellularly and in plasma. Furthermore, PRKCB2 and SOCS3 mRNA were also related to obesity (BMI) and

Table 2 Levels of pro-inflammatory markers/mediators (means \pm SE)

\begin{tabular}{lll}
\hline & Lean subjects & Obese subjects \\
\hline NFKB binding activity $^{\mathrm{a}}$ & $9,415 \pm 2,354$ & $24,935 \pm 2,874^{*}$ \\
TNFAIP1 $\mathrm{mRNA}^{\mathrm{a}}$ & $0.545 \pm 0.145$ & $0.939 \pm 0.268^{*}$ \\
IL6 $\mathrm{mRNA}^{\mathrm{a}}$ & $0.339 \pm 0.084$ & $0.777 \pm 0.262^{*}$ \\
TNF $\alpha(\mathrm{pg} / \mathrm{ml})$ & $2.772 \pm 0.311$ & $3.753 \pm 0.621^{*}$ \\
IL6 $(\mathrm{pg} / \mathrm{ml})$ & $1.445 \pm 0.423$ & $3.297 \pm 0.895^{*}$ \\
CRP $(\mathrm{ng} / \mathrm{ml})$ & $842 \pm 125$ & $3,041 \pm 654^{*}$ \\
\hline
\end{tabular}

${ }^{\mathrm{a}}$ Arbitrary units

$n=16$ in each group

${ }^{*} p<0.05$

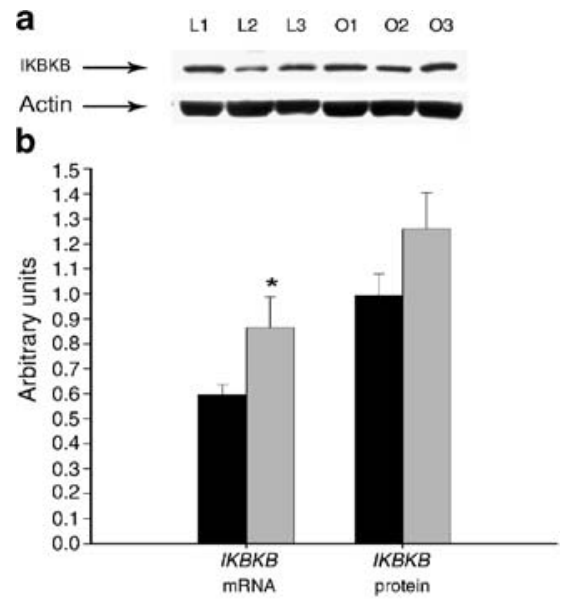

Fig. 3 a Protein content of IKBKB from total cell lysates of three lean $(L)$ and three obese $(O)$ subjects. b mRNA expression (normalised to $18 \mathrm{~S}$ ) and densitometry of protein content of IKBKB normalised to actin ( $n=16$ vs 16 ). Grey bars, obese subjects; black bars, lean subjects. $* p<0.05$

NEFA concentrations. IKBKB mRNA was related to NFKB binding, TNFAIP1 and SOCS3 mRNA, while IKBKB protein was related to $S O C S 3$ mRNA.

It is noteworthy that SOCS3 mRNA was negatively related to $p$-INSR- $\beta$ and $p$-INSR- $\beta$ : INSR- $\beta$ ratio while PRKCB2 showed only a trend towards this relationship. It is, therefore, relevant that SOCS3 mRNA was also related to HOMA-IR, an index of systemic insulin resistance. The mRNA expression of other PRKC isoforms ( $B 1, Q$ and $Z$ ) was not significantly elevated in the MNC from the obese subjects, nor were they related to BMI or HOMA-IR. IKBKB was related to PRKCB2 mRNA and BMI, but not to $p$-INSR- $\beta$ or $p$-INSR- $\beta$ : INSR- $\beta$ ratio or HOMA-IR.

Thus, SOCS3 and possibly PRKCB2, may contribute to the diminution in insulin signalling through an interference with insulin signal transduction and thus induce a state of insulin resistance in the MNC. We have previously demonstrated that insulin has a comprehensive anti-inflammatory effect on peripheral blood MNCs [6, 7]. This is reflected in the suppression of reactive oxygen species generation and NFKB binding, an increase in I $\mathrm{KB} \alpha$ production, and a suppression of EGR1 binding and production. Consistent with an insulin resistant state of this cell in obesity, we have previously shown that MNCs exist in a pro-inflammatory state [9]. The pro-inflammatory state in the obese is also in part due to excessive macronutrient intake, which induces oxidative stress as well as inflammation, including increased $\mathrm{NF \kappa B}$ binding [23]. It is thus relevant that we have recently observed that the intake of a fatty meal also induces an increase in SOCS3 mRNA expression (unpublished observations). The increase in SOCS3 mRNA expression may also contribute to leptin 


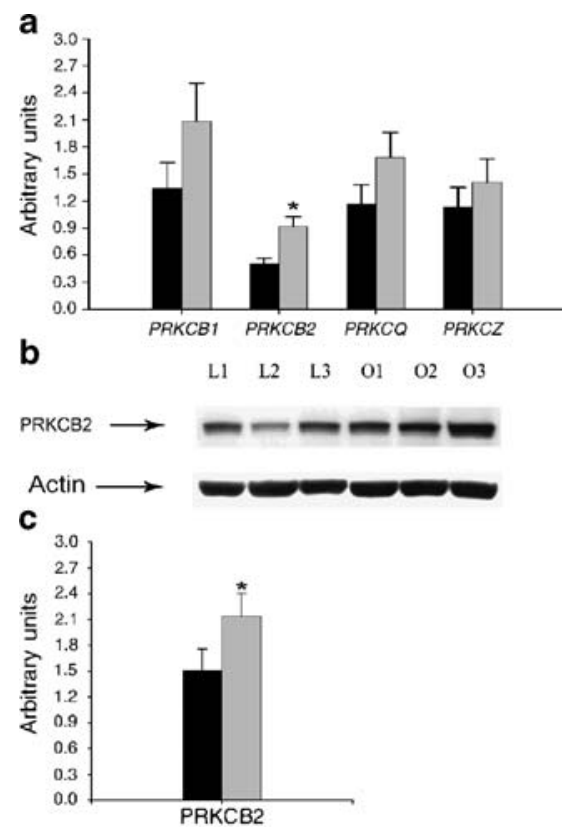

Fig. 4 a mRNA expression of $P R K C B 1, P R K C B 2, P R K C Q$ and $P R K C Z$ in MNCs from obese and lean subjects measured by real-time RT-PCR. Values are normalised to $18 \mathrm{~S}$ expression ( $n=16$ vs 16 ). $* p<0.01$. b Protein content of PRKCB2 from total cell lysates of three lean $(L)$ and three obese $(O)$ subjects. c Densitometry of protein content of PRKCB2 ( $n=16$ vs 16). ${ }^{*} p<0.05$. Grey bars, obese subjects; black bars, lean subjects

resistance since SOCS3 also interferes with leptin signalling and the Janus kinase and the signal transducer and activator of transcription (JAK-STAT) pathway.

While our data show that there is a diminution in p-INSR$\beta$ and the pINSR- $\beta$ : INSR- $\beta$ ratio, consistent with impaired insulin signalling, it is also of interest that the p-INSR- $\beta$ : INSR $-\beta$ ratio in MNC was significantly related to HOMA-IR $(r=0.5, p<0.005)$. This suggests that there may be a relationship between the pro-inflammatory insulin resistant state of the MNC, insulin signalling mechanisms and the general metabolic state of insulin resistance as reflected in HOMA-IR. These changes may also contribute to leptin resistance known to occur in the obese.

The MNC is not a classic insulin-responsive metabolic cell. However, it was the first cell shown to have INSRs. Our recent work has shown that insulin may exert an antiinflammatory effect on both the $\mathrm{MNC}$, in vivo, and the endothelial cell, in vitro. More recently, the monocyte in the $\mathrm{MNC}$ fraction has been shown to migrate to adipose tissue and to induce inflammatory responses, which in turn leads to cytokine production by adipocytes which may mediate insulin resistance $[24,25]$. Indeed, in a recent seminal experiment, the deletion of IKBKB, a kinase which phosphorylates IKB to cause its phosphorylation, ubiquitination and proteolysis, and thus the activation of the proinflammatory transcription factor NFkB, led not only to an

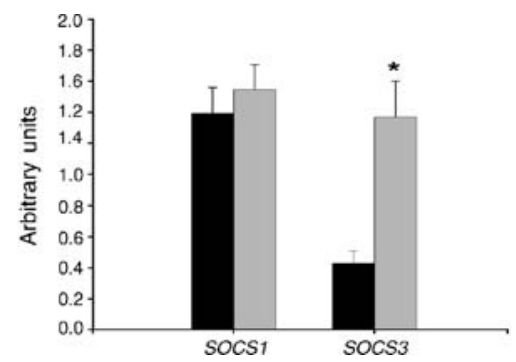

Fig. 5 mRNA expression of SOCS1 and SOCS3 in the MNC from obese (grey bars) and lean (black bars) subjects measured by real-time RT-PCR. Values are normalised to $18 \mathrm{~S}$ expression ( $n=16$ vs 16$) .{ }^{*} p<$ 0.001

anti-inflammatory state but a protection from systemic insulin resistance induced by a high-fat diet [26]. Thus, the myeloid-derived monocyte-macrophages play a key role in the pathogenesis of insulin resistance. Furthermore, this role in the pathogenesis of insulin resistance is dependent upon inflammatory mechanisms, since the deletion of IKBKB leads to a protection from systemic insulin resistance. These facts are even more intriguing since the deletion of IKBKB from hepatocytes or adipoctyes leads to protection from insulin resistance of only that specific organ but not of other organs, unlike the deletion of IKBKB from myelogenous leucocytes, which provided systemic insulin sensitisation. These data attribute a key role to the myelogenous leucocytes in the pathogenesis of insulin resistance.

It is thus relevant that $I K B K B$ expression is increased at both mRNA and protein levels in MNCs from the obese as observed in our experiments. The monocyte, which is an important component of MNCs, thus expresses an excess of the gene whose deletion in the mouse protects from insulin resistance systemically. It is possible that the production of IKBKB in the MNC may contribute significantly to the state of insulin resistance systemically by modulating inflammatory mechanisms and not by interfering with insulin signalling directly. The role of the monocyte may be mediated through its ability to infiltrate into various organs including the adipose tissue, the skeletal muscle and the liver.

The most impressive relationship of $I K B K B$ mRNA and protein is with SOCS3 mRNA and PRKCB2 mRNA in addition to IL6 in plasma. It is possible that while IL6 drives SOCS3, which in turn interferes with insulin signal transduction, PRKCB2 mRNA, may also contribute to IKBKB phosphorylation and activation. These issues need to be analysed in a dynamic experiment following insulin stimulation. In the meantime our data provide the first evidence in terms of the relationships between IKBKB mRNA and protein production, PRKCB2 mRNA and protein and SOCS3 mRNA expression in the commonest 
cause of insulin resistance, the state of obesity. It is of interest that IKBKB protein and IKBKB mRNA are not related to each other. It would appear that there are specific regulators of IKBKB in terms of its translation and its proteasomal degradation.

Experimental evidence in adipocytes, in vitro, demonstrates that SOCS3 may bind to the tyrosine phosphorylated INSR and therefore interfere with INSR-induced tyrosine phosphorylation of INSRS-1 [16]. SOCS3 may also cause ubiquitination and proteasomal degradation of INSRS-1 and INSRS-2 in hepatocytes [27]. These two mechanisms have been proposed as a possible mode of action of SOCS3. The fact that INSR tyrosine phosphorylation is reduced in the obese with no change in INSRS-1 quantity suggests that SOCS3 in the MNC might interfere with insulin signalling through a direct inhibition of p-INSR$\beta$ but not through the ubiquitination or the degradation of INSRS-1. SOCS3 has also been implicated in the reduction of leptin signal transduction through an interference with the JAK-STAT pathway. It may therefore, also play a role in the pathogenesis in leptin resistance in obesity [28-30]. MNC (monocytes and lymphocytes) are known to express the leptin receptor.

Since plasma NEFA concentrations are thought to modulate insulin resistance, we examined the relationship of NEFA to BMI and HOMA-IR; there was a significant correlation between NEFA and BMI but there was only a statistical trend relating NEFA and HOMA-IR. There was also no relationship between NEFA and p-INSR- $\beta$ and the $p$ INSR- $\beta$ : INSR- $\beta$ ratio (ESM Table 1 ). On the other hand, NEFA concentrations were highly significantly related to inflammatory mediators such as CRP, IL6 and TNFAIP1, and to SOCS3 mRNA. It is possible that in obesity, NEFA induces insulin resistance by inducing inflammation and that an inflammatory mediator interferes with insulin signalling. We have recently demonstrated that an infusion of triacylglycerol and heparin to induce an increase in NEFA concentrations to levels comparable with those in the obese induces an acute increase in reactive oxygen species generation and $\mathrm{NFKB}$ binding by MNCs in normal subjects [31], while inducing insulin resistance. The above conclusions are based on statistical associations and are, therefore, at this stage only suggestive but not definitive.

This study links inflammatory mediators, PRKCB2, SOCS3 and IKBKB, firmly to insulin resistance, which is a risk factor for atherosclerosis. It thus provides the basis for the 'common soil hypothesis' [32], which provides a link between insulin resistant states of obesity and type 2 diabetes and atherosclerosis.

In conclusion, obesity is associated with a significant reduction in pINSR- $\beta$ in MNCs, consistent with a reduction in INSR activation. The increases in NFKB binding, TNFAIP1 mRNA expression and plasma CRP concentrations have a significant inverse relationship with pINSR. The increased levels of PRKCB2 is related to inflammatory mediators but not to $\mathrm{p}$-INSR- $\beta$. The elevation in SOCS3 mRNA expression was inversely related to INSR phosphorylation and directly related to inflammatory mediators. Thus while PRKCB2 drives inflammation, it probably does not interfere with INSR phosphorylation directly. On the other hand, SOCS3 is induced by and is related to inflammatory mediators and probably interferes with INSR phosphorylation. It may thus contribute to insulin and leptin resistance in the obese (Fig. 6).

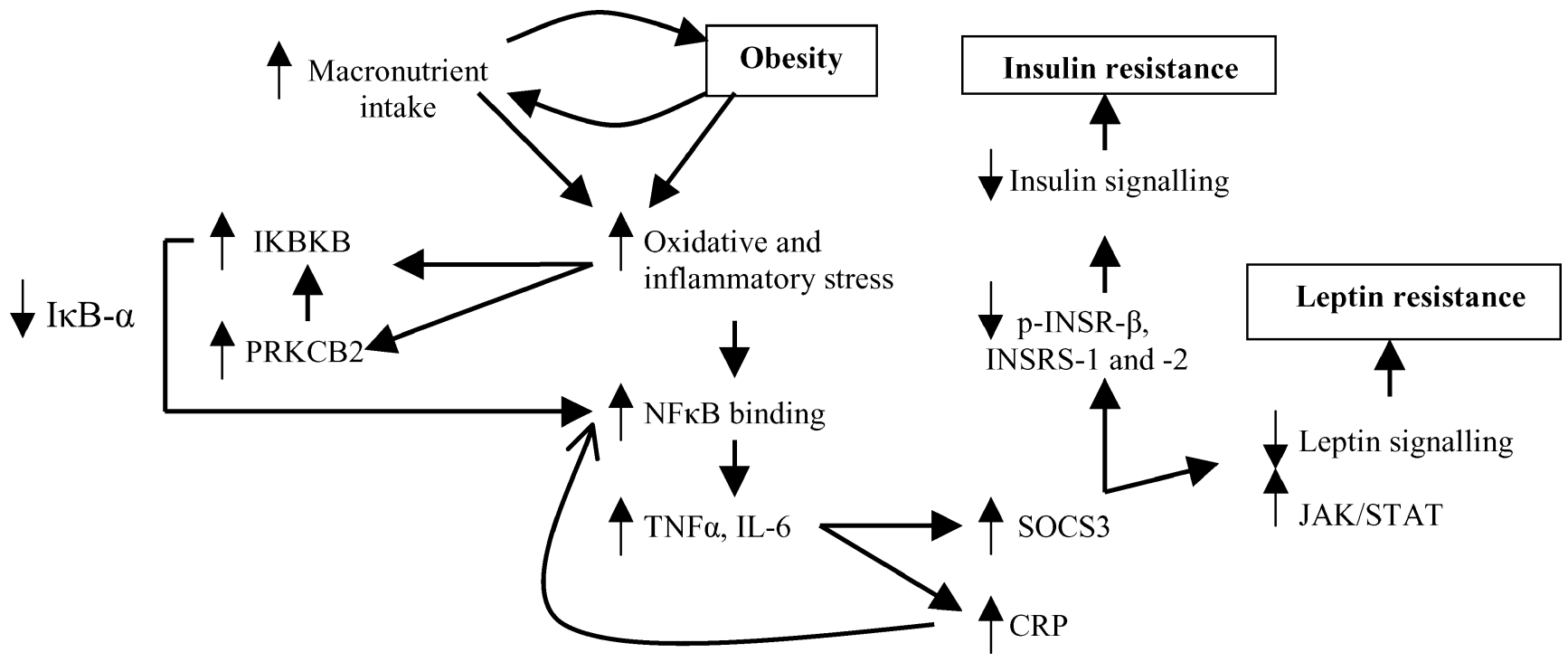

Fig. 6 Schematic representation of the possible interactions of obesity, inflammation and insulin resistance. Obesity-mediated inflammatory mediators such as SOCS3 might interfere with insulin signalling in the MNC and other tissues 
Duality of interest The authors did not receive any financial support to conduct this study.

\section{References}

1. Hotamisligil GS, Shargill NS, Spiegelman BM (1993) Adipose expression of tumor necrosis factor-alpha: direct role in obesitylinked insulin resistance. Science 259:87-91

2. Hotamisligil GS, Peraldi P, Budavari A, Ellis R, White MF, Spiegelman BM (1996) IRS-1-mediated inhibition of insulin receptor tyrosine kinase activity in TNF-alpha- and obesityinduced insulin resistance. Science 271:665-668

3. Hotamisligil GS, Murray DL, Choy LN, Spiegelman BM (1994) Tumor necrosis factor alpha inhibits signaling from the insulin receptor. Proc Natl Acad Sci USA 91:4854-4858

4. Hotamisligil GS, Arner P, Caro JF, Atkinson RL, Spiegelman BM (1995) Increased adipose tissue expression of tumor necrosis factor-alpha in human obesity and insulin resistance. J Clin Invest 95:2409-2415

5. Aljada A, Ghanim H, Assian E, Dandona P (2002) Tumor necrosis factor-alpha inhibits insulin-induced increase in endothelial nitric oxide synthase and reduces insulin receptor content and phosphorylation in human aortic endothelial cells. Metabolism 51:487-491

6. Dandona P, Aljada A, Mohanty P et al (2001) Insulin inhibits intranuclear nuclear factor kappaB and stimulates IkappaB in mononuclear cells in obese subjects: evidence for an antiinflammatory effect? J Clin Endocrinol Metab 86:3257-3265

7. Aljada A, Ghanim H, Mohanty P, Kapur N, Dandona P (2002) Insulin inhibits the pro-inflammatory transcription factor early growth response gene-1 (Egr)-1 expression in mononuclear cells (MNC) and reduces plasma tissue factor (TF) and plasminogen activator inhibitor-1 (PAI-1) concentrations. J Clin Endocrinol Metab 87:1419-1422

8. Gavin JR 3rd, Roth J, Jen P, Freychet P (1972) Insulin receptors in human circulating cells and fibroblasts. Proc Natl Acad Sci USA 69:747-751

9. Ghanim H, Aljada A, Hofmeyer D, Syed T, Mohanty P, Dandona $\mathrm{P}$ (2004) Circulating mononuclear cells in the obese are in a proinflammatory state. Circulation 110:1564-1571

10. Dandona P, Weinstock R, Thusu K, Abdel-Rahman E, Aljada A, Wadden T (1998) Tumor necrosis factor-alpha in sera of obese patients: fall with weight loss. J Clin Endocrinol Metab 83:29072910

11. Bistrian BR, Khaodhiar L (2000) Chronic systemic inflammation in overweight and obese adults. JAMA 283:2235, Author reply 2236

12. Bastard JP, Jardel C, Delattre J, Hainque B, Bruckert E, Oberlin F (1999) Evidence for a link between adipose tissue interleukin-6 content and serum C-reactive protein concentrations in obese subjects. Circulation 99:2221-2222

13. Chin JE, Dickens M, Tavare JM, Roth RA (1993) Overexpression of protein kinase $\mathrm{C}$ isoenzymes alpha, beta I, gamma, and epsilon in cells overexpressing the insulin receptor. Effects on receptor phosphorylation and signaling. J Biol Chem 268:6338-6347

14. Bossenmaier B, Mosthaf L, Mischak H, Ullrich A, Haring HU (1997) Protein kinase C isoforms beta 1 and beta 2 inhibit the tyrosine kinase activity of the insulin receptor. Diabetologia 40:863-866
15. Itani SI, Zhou Q, Pories WJ, MacDonald KG, Dohm GL (2000) Involvement of protein kinase $\mathrm{C}$ in human skeletal muscle insulin resistance and obesity. Diabetes 49:1353-1358

16. Emanuelli B, Peraldi P, Filloux C et al (2001) SOCS-3 inhibits insulin signaling and is up-regulated in response to tumor necrosis factor-alpha in the adipose tissue of obese mice. J Biol Chem 276:47944-47949

17. Emanuelli B, Peraldi P, Filloux C, Sawka-Verhelle D, Hilton D, Van Obberghen E (2000) SOCS-3 is an insulin-induced negative regulator of insulin signaling. J Biol Chem 275:15985-15991

18. Mooney RA, Senn J, Cameron S et al (2001) Suppressors of cytokine signaling- 1 and -6 associate with and inhibit the insulin receptor. A potential mechanism for cytokine-mediated insulin resistance. J Biol Chem 276:25889-25893

19. Matthews DR, Hosker JP, Rudenski AS, Naylor BA, Treacher DF, Turner RC (1985) Homeostasis model assessment: insulin resistance and beta-cell function from fasting plasma glucose and insulin concentrations in man. Diabetologia 28:412-419

20. Chomczynski P, Sacchi N (1987) Single-step method of RNA isolation by acid guanidinium thiocyanate-phenol-chloroform extraction. Anal Biochem 162:156-159

21. Andrews NC, Faller DV (1991) A rapid micropreparation technique for extraction of DNA-binding proteins from limiting numbers of mammalian cells. Nucleic Acids Res 19:2499

22. Sasaki A, Inagaki-Ohara K, Yoshida T et al (2003) The N-terminal truncated isoform of SOCS3 translated from an alternative initiation AUG codon under stress conditions is stable due to the lack of a major ubiquitination site, Lys-6. J Biol Chem 278:2432-2436

23. Aljada A, Mohanty $P$, Ghanim $H$ et al (2004) Increase in intranuclear nuclear factor kappaB and decrease in inhibitor kappaB in mononuclear cells after a mixed meal: evidence for a proinflammatory effect. Am J Clin Nutr 79:682-690

24. Weisberg SP, McCann D, Desai M, Rosenbaum M, Leibel RL, Ferrante AW Jr (2003) Obesity is associated with macrophage accumulation in adipose tissue. J Clin Invest 112:1796-1808

25. Xu H, Barnes GT, Yang Q et al (2003) Chronic inflammation in fat plays a crucial role in the development of obesity-related insulin resistance. J Clin Invest 112:1821-1830

26. Arkan MC, Hevener AL, Greten FR et al (2005) IKK-beta links inflammation to obesity-induced insulin resistance. Nat Med 11:191-198

27. Rui L, Yuan M, Frantz D, Shoelson S, White MF (2002) SOCS-1 and SOCS-3 block insulin signaling by ubiquitinmediated degradation of IRS1 and IRS2. J Biol Chem 277: 42394-42398

28. Bjorbaek C, El-Haschimi K, Frantz JD, Flier JS (1999) The role of SOCS-3 in leptin signaling and leptin resistance. J Biol Chem 274:30059-30065

29. Bjorbaek C, Elmquist JK, Frantz JD, Shoelson SE, Flier JS (1998) Identification of SOCS-3 as a potential mediator of central leptin resistance. Mol Cell 1:619-625

30. Howard JK, Cave BJ, Oksanen LJ, Tzameli I, Bjorbaek C, Flier JS (2004) Enhanced leptin sensitivity and attenuation of dietinduced obesity in mice with haploinsufficiency of Socs3. Nat Med 10:734-738

31. Tripathy D, Mohanty P, Dhindsa S et al (2003) Elevation of free fatty acids induces inflammation and impairs vascular reactivity in healthy subjects. Diabetes 52:2882-2887

32. Stern MP (1995) Diabetes and cardiovascular disease. The 'common soil' hypothesis. Diabetes 44:369-374 